Review

\title{
Mechanism of ribosome translation through mRNA secondary structures
}

\author{
Ping $\mathrm{Xie}^{1,2 \llbracket}$, Hong Chen ${ }^{1}$ \\ 1. School of Materials Science and Energy Engineering, FoShan University, Guangdong, 528000, China; \\ 2. Key Laboratory of Soft Matter Physics and Beijing National Laboratory for Condensed Matter Physics, Institute of Physics, Chinese Academy of Sciences, \\ Beijing 100190, China. \\ $\triangle$ Corresponding author: pxie@aphy.iphy.ac.cn \\ (c) Ivyspring International Publisher. This is an open access article distributed under the terms of the Creative Commons Attribution (CC BY-NC) license \\ (https://creativecommons.org/licenses/by-nc/4.0/). See http://ivyspring.com/terms for full terms and conditions.
}

Received: 2017.02.06; Accepted: 2017.03.07; Published: 2017.05.16

\begin{abstract}
A ribosome is a macromolecular machine that is responsible for translating the genetic codes in messenger RNA (mRNA) into polypeptide chains. It has been determined that besides translating through the single-stranded region, the ribosome can also translate through the duplex region of mRNA by unwinding the duplex. To understand the mechanism of ribosome translation through the duplex, several models have been proposed to study the dynamics of mRNA unwinding. Here, we present a comprehensive review of these models and also discuss other possible models. We evaluate each model and discuss the consistency and/or inconsistency between the theoretical results that are obtained based on each model and the available experimental data, thus determining which model is the most reasonable one to describe the mRNA unwinding mechanism and dynamics of the ribosome. Moreover, a framework for future studies in this subject is provided.
\end{abstract}

Key words: Protein synthesis; translocation; tRNA; mRNA; ribosomal rotation; molecular machine.

\section{Introduction}

A ribosome is a macromolecular machine responsible for the synthesis of protein chains by a process called translation, where the sequence of the amino acid residues is encoded by that of codons in messenger RNA (mRNA). During translation, any secondary structure present in the downstream mRNA must be disrupted for the ribosome to read the codon in the single-stranded mRNA. Biochemical studies by Takyar et al. [1] firstly revealed that the mRNA duplex can be unwound by the ribosome itself, without the requirement of exogenous RNA helicases. Then, the dynamics of mRNA unwinding by the ribosome has been studied extensively by employing different single-molecule methods such as the single-molecule optical trapping (smOT) and single-molecule fluorescence resonance energy transfer (smFRET). With smOT (see Fig. 1), Wen et al. [2] firstly followed translation through the mRNA duplex by single ribosomes in real time and studied the distribution of dwell times in the translation elongation cycle. Later, with smOT (see Fig. 1) the dependence of the translation rate on the force applied to the ends of the mRNA duplex to unzip the duplex was elaborately determined by $\mathrm{Qu}$ et al. [3]. With smFRET by monitoring efficiencies of FRET between the two tRNAs bound to the ribosome, FRET between tRNA and L11 near the A site, and FRET between tRNA and L1 stalk near the E-site (see Fig. 1), Chen et al. [4] studied the effect of downstream mRNA secondary structures on tRNA translocation in the 50S subunit and deacylated tRNA dissociation from the E/E site. With smFRET by monitoring the efficiency of FRET between the L1 stalk and tRNA (L1-tRNA FRET) (see Fig. 1), Kim et al. [5] revealed that that when the ribosome translates through the mRNA containing the downstream stem loop, even in the presence of EF-G of high concentration $(1 \mu \mathrm{M})$ the L1-tRNA FRET efficiency exhibits multiple fluctuations between a low $(\sim 0.2)$ and high $(\sim 0.8)$ values before undergoing mRNA translocation (see Fig. 1), and by contrast, when the ribosome translates through the mRNA lacking the stem loop, the 
L1-tRNA FRET efficiency exhibits the high value $(\sim 0.8)$ approximately once before undergoing mRNA translocation (see Fig. 1). In addition, with a force-induced remnant magnetization spectroscopy technique, the ribosomal translocation through DNA-RNA hybrid was also investigated [6]. However, the molecular mechanism of translation through the mRNA secondary structure by the ribosome remains unclear.

To understand the mechanism of mRNA unwinding and explain the smOT data $[2,3]$, three models have been proposed up to now $[3,7,8]$. Here, we present a brief review of the three models and moreover, consider other five models. Each model is evaluated and the consistency and/or inconsistency between the theoretical results that are obtained based on each model and the available experimental data are discussed, thus determining which one is most reasonable.

\section{Model I}

The smOT data showed that the rate of ribosome translation through the mRNA duplex is reduced relative to that of translation through the single-stranded mRNA [2,3]. The simplest model (called Model I) to explain this reduction of translation rate is shown in Fig. 1, where the reduced translation rate arises from the reduction of the rate constant of the mRNA translocation step, which is induced by the resistance force resulting from the unwinding of the downstream mRNA base pairs to impede the translocation.
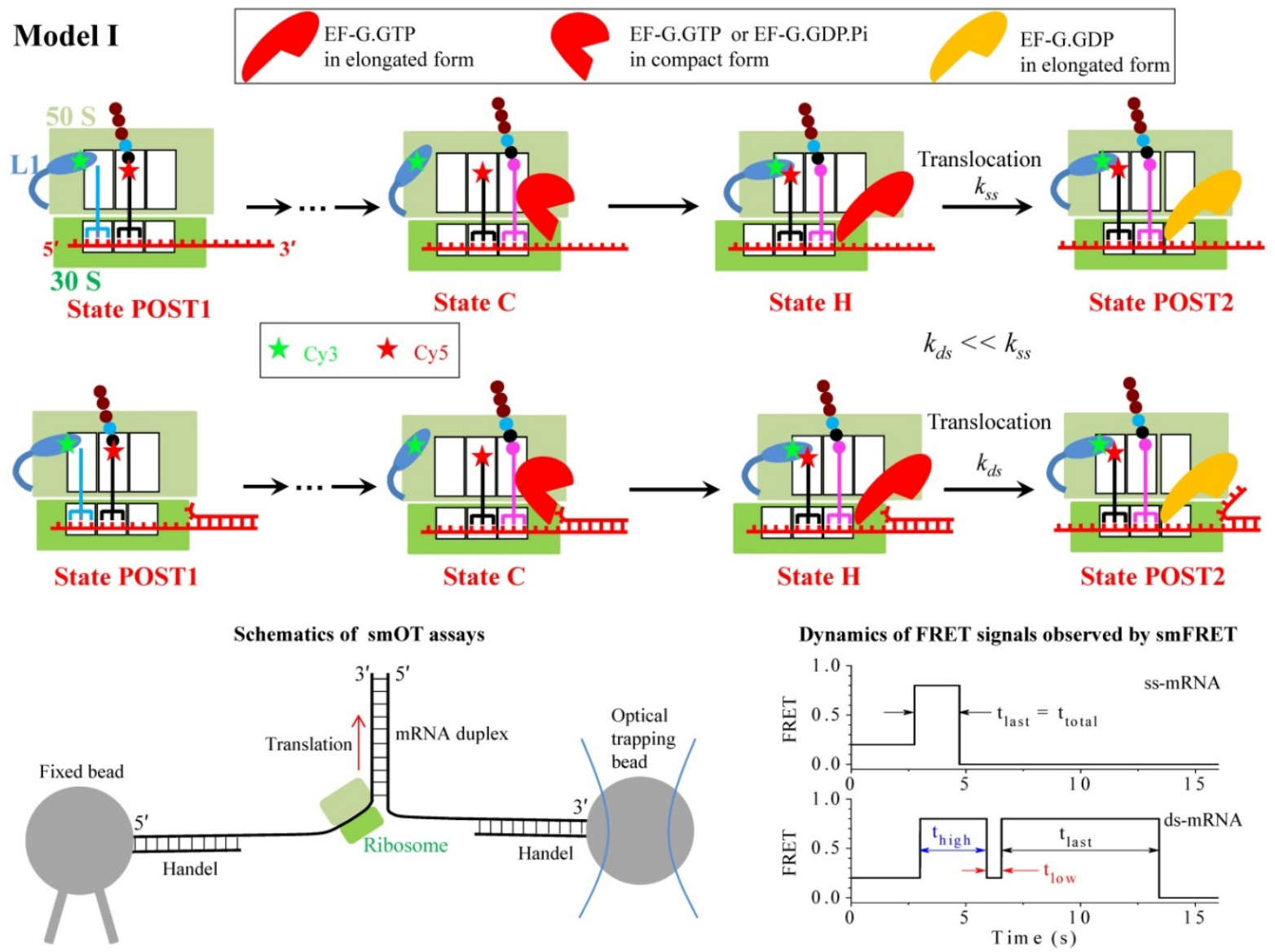

Figure 1. Schematic representation of Model I (upper and middle panels). From the posttranslocation state (State POST1), after rapid deacylated tRNA dissociation, the aminoacyl-tRNA.EF-Tu.GTP ternary complex binding in the A/T state, GTP hydrolysis, Pi and EF-Tu.GDP releases, the accommodation of the aminoacyl-tRNA into its fully bound $\mathrm{A} / \mathrm{A}$ state and then the peptidyl transfer, the ribosomal complex transits to the classical non-rotated pretranslocation state. EF-G.GTP of saturating concentration binds immediately, facilitating the classical non-rotated pretranslocation state (State C) transition to rotated or hybrid state, and GTP hydrolysis to GDP.Pi in hybrid state induces the ribosomal unlocking, opening the mRNA channel (State $\mathrm{H}$ ). The subsequent reverse intersubunit rotation causes the small ribosomal $30 \mathrm{~S}$ subunit translocating forward by unwinding three mRNA base pairs, with State $\mathrm{H}$ transiting to State POST2, from which the next elongation cycle continues. Upper panels show the translation through the single-stranded mRNA, with the rate constant of the translocation step being denoted by $k_{s s}$, while the middle panels show the translation through the mRNA duplex, with the rate constant of the translocation step being denoted by $k_{d s}$. The resistance force resulting from the unwinding of the downstream three mRNA base pairs impedes the translocation, reducing the rate constant of the translocation step, with $k_{d s}<<k_{s s}$. Schematics of the Cy3-labeled L1 stalk and Cy5-labeled tRNA in smFRET experiments [4, 5] is shown. The bottom left panel represents the schematics of the smOT experiments used to study mRNA structure unwinding by the ribosome [2, 3]. The bottom right panel show schematically representative time traces of LI-tRNA FRET for the mRNA lacking the downstream secondary structure (ss-mRNA) and for the mRNA containing the downstream secondary structure (ds-mRNA) observed in smFRET experiments at saturating EF-G.GTP [5], with the measured mean values, $\tau_{\text {last }}=\left\langle t_{\text {last }}>\approx 0.6 \mathrm{~s}\right.$ for the former case, and $\tau_{\text {high }}=\left\langle t_{\text {high }}\right\rangle \approx 2.1 \mathrm{~s}, \tau_{\text {low }}=\left\langle t_{\text {low }}>\approx 0.55 \mathrm{~s}\right.$ and $\tau_{\text {last }}=\left\langle t_{\text {last }}\right\rangle \approx 6.4 \mathrm{~s}$ for the latter case. 
Model I indicates that no matter when translation through the mRNA secondary structure or translation through the single-stranded mRNA, the ribosomal complex samples the hybrid state (State $\mathrm{H}$ ) only once before undergoing mRNA translocation at saturating concentrations (e.g., $1 \mu \mathrm{M}$ ) of EF-G.GTP (the smFRET data showed that the translocation time decreases with increasing EF-G.GTP concentrations up to $0.5 \mu \mathrm{M}$ and levels [5], and the smOT data showed that the translation rate becomes saturating at EF-G.GTP concentrations larger than $0.2 \mu \mathrm{M}$ [3]), but with the lifetime of the hybrid state being longer for the former than for the latter. This implies that at saturating EF-G.GTP the L1-tRNA FRET efficiency shows the high value $(\sim 0.8)$ only once before undergoing mRNA translocation (noting that after the mRNA translocation deacylated tRNA dissociates from the E/E site rapidly [9]). This is inconsistent with the smFRET data of Kim et al. [5] showing that at EF-G.GTP concentration of $1 \mu \mathrm{M}$, when translating through the mRNA duplex the ribosomal complex exhibits multiple fluctuations between low $(\sim 0.2)$ and high ( 0.8) L1-tRNA FRET efficiencies before undergoing mRNA translocation whereas when translating through the single-stranded mRNA the complex samples the high ( 0.8) FRET value approximately only once before undergoing mRNA translocation. Alternatively, it may be argued that during multiple fluctuations the transition from the hybrid state to classical non-rotated state occurs after the dissociation of EF-G but before the re-binding of
EF-G. As analyzed before [10], based on this argument the obtained theoretical data on the dependence of the number of the transition from the hybrid to classical non-rotated state on EF-G.GTP concentration are deviated significantly away from the smFRET data [5]. Additionally, as studies showed [11], based on the consideration that the reduced translation rate arises from the reduction of the rate constant of the mRNA translocation step, the theoretical data on dwell-time distribution of translation through the mRNA duplex versus the external force to unwind the duplex are deviated significantly away from the available smOT data [2]. Thus, Model I is not a reasonable one to explain the reduced rate of ribosome translation through the mRNA secondary structure.

\section{Model II}

To quantitatively explain their smOT data, Qu et al. [3] proposed a model (called Model II) (Fig. 2), in which the ribosome uses two active mechanisms to promote mRNA unwinding: open-state stabilization and mechanical unwinding. In the first mechanism, the ribosome interacts preferentially with the open form of the junction, favoring the open state in the thermal fluctuations between the open and closed states and thus increasing the probability of the ribosome translocating unhindered. In the second mechanism, when the ribosome encounters the junction in the closed state, it breaks the junction and translocates forward.
Model II

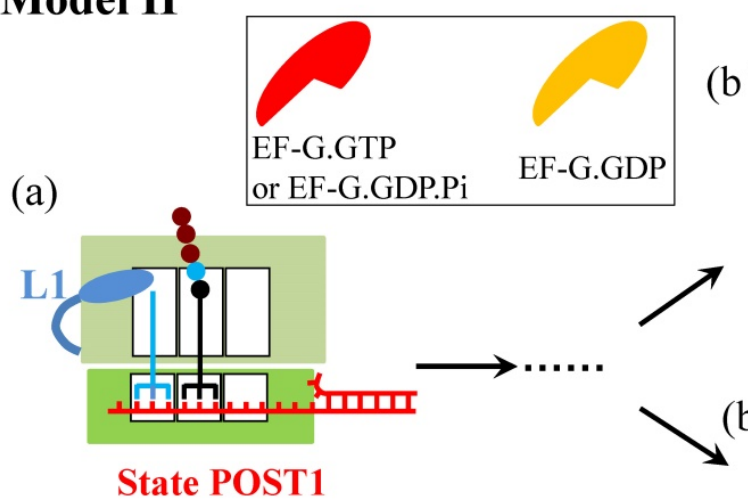

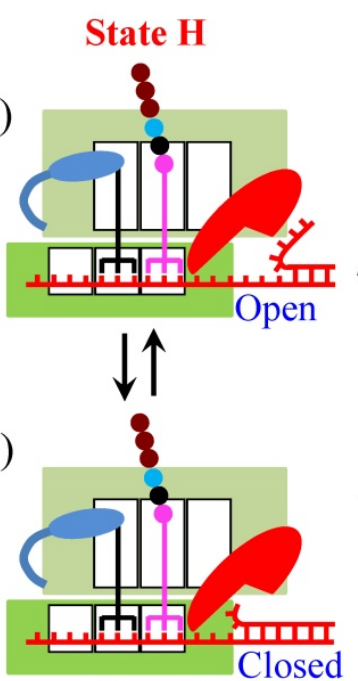

State $\mathbf{H}$

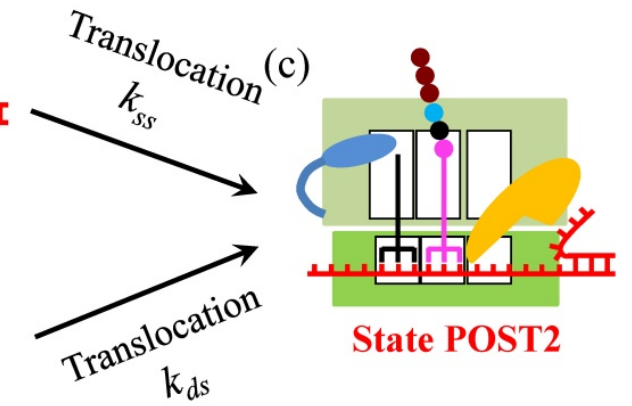

Figure 2. Schematic representation of Model II [3]. The transitions from State POST1 through State H are the same as those in Fig. 1. From State H, the subsequent reverse intersubunit rotation causes the small ribosomal 30 S subunit translocating forward and unwinding mRNA duplex with two active mechanisms. In the first mechanism (from b' to c), the ribosome interacts preferentially with the open form of the mRNA junction and then translocats unhindered, becoming State POST2. In the second mechanism (from $b$ to $c$ ), the ribosome breaks the mRNA duplex and translocates forward, becoming State POST2.

Similar to Model I, Model II also indicates that no matter when translation through the mRNA 
secondary structure or translation through the single-stranded mRNA, at saturating EF-G.GTP the ribosomal complex samples the hybrid state (State $\mathrm{H}$ ) only once before undergoing mRNA translocation, which is inconsistent with the smFRET data of Kim et al. [5]. In addition, recent experiments on -1 frameshifting (FS) efficiency and stability of the mRNA pseudoknot that affects the -1 FS showed no correlation between the -1 FS efficiency and the thermal stability of the pseudoknot, and by contrast, a positive correlation between the -1 FS efficiency and the unfolding force or an inverse correlation between the -1 FS efficiency and the mechanical unfolding rate [12]. These results indicate evidently that the ribosome is a force-generating molecular motor with helicase activity [12], arguing against the first mechanism of Model II.

\section{Model III}

A model (called Model III) (Fig. 3) was proposed recently by assuming that besides affecting the translocation step, the downstream mRNA duplex also affects the rate constant of aminoacyl-tRNA accommodation step [8]. In Model III, the reduction of translation rate arises from both the reduction of the rate constant of the mRNA translocation step and that of the aminoacyl-tRNA accommodation step.

Similar to Model I and Model II, Model III also indicates that no matter when translation through the mRNA secondary structure or translation through the single-stranded mRNA, at saturating EF-G.GTP the ribosomal complex samples the hybrid state (State $\mathrm{H}$ ) only once before undergoing mRNA translocation, which is inconsistent with the smFRET data of Kim et al. [5].

Moreover, the assumption that the downstream mRNA duplex reduces the rate constant of aminoacyl-tRNA accommodation step is also inconsistent with the smFRET data [5]. As summarized in Shakiba et al. [8], the available biochemical data showed that the aminoacyl-tRNA accommodation is the rate-limiting step in the process of the binding of the aminoacyl-tRNA.EF-Tu.GTP ternary complex of saturating concentration, aminoacyl-tRNA accommodation, peptidyl transfer and the first conformational transition to the hybrid state. It is thus expected that the time duration between the delivery of the ternary complex and the first transition to the high ( 0.8) L1-tRNA FRET efficiency (corresponding to the hybrid state) from the low ( 0.2) FRET efficiency (corresponding to the non-rotated state) for the case of translation through the mRNA duplex would be larger than that for the case of translation through the single-stranded mRNA. This is contrary to the smFRET data showing that the durations for the two cases are nearly the same (even with the former being slightly smaller than the latter) [5]. On the contrary, the smFRET data indicate that the downstream mRNA duplex has little effect on the rate constant of aminoacyl-tRNA accommodation.

\section{Model III}

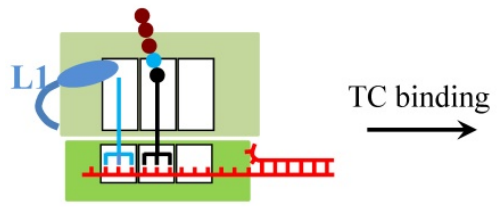

State POST1

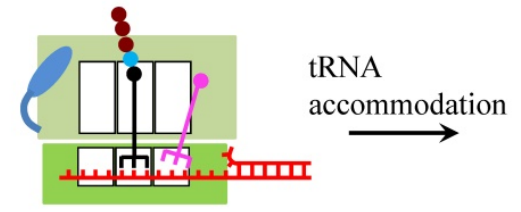

State A/T

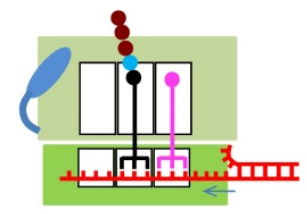

State A/A

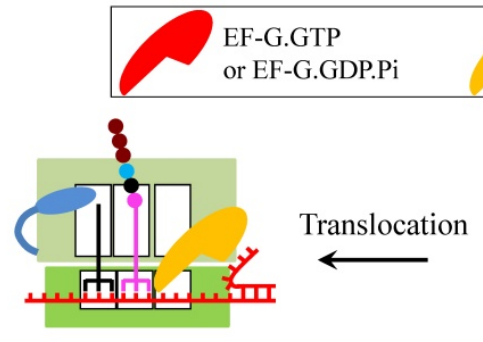

State POST2
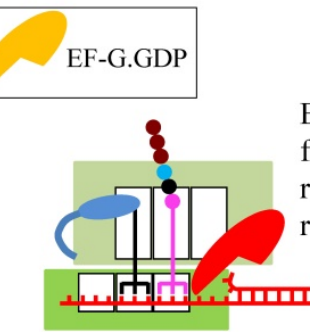

State $\mathbf{H}$

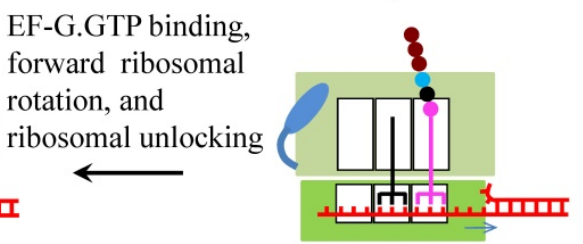

State C

Figure 3. Schematic representation of Model III [8]. From the posttranslocation state (State POST1), deacylated tRNA dissociates rapidly and the aminoacyl-tRNA.EF-Tu.GTP ternary complex binds in the initial A/T state (State A/T). Upon accommodation of the aminoacyl-tRNA (State A/A), the tRNA of the A site is twisted, and as a result, the anticodon and the associated mRNA are displaced by $9 \AA$ into the entry channel of the ribosome, unwinding an mRNA base pair. After the peptidyl transfer (State C), the stress in the mRNA is released. Then, EF-G.GTP of saturating concentration binds immediately, facilitating the classical non-rotated pretranslocation state transition to hybrid state. GTP hydrolysis to GDP.Pi in hybrid state induces the ribosomal unlocking, opening the mRNA channel $($ State $\mathrm{H})$. The subsequent reverse intersubunit rotation causes the small ribosomal $30 \mathrm{~S}$ subunit translocating forward by unwinding three mRNA base pairs, with State $\mathrm{H}$ transiting to State POST2, from which the next elongation cycle continues. 


\section{Model IV}

(a)

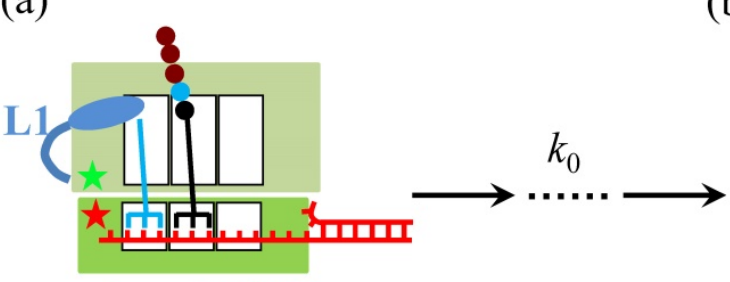

State POST1 (b)

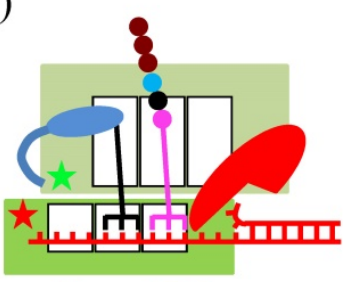

State H1

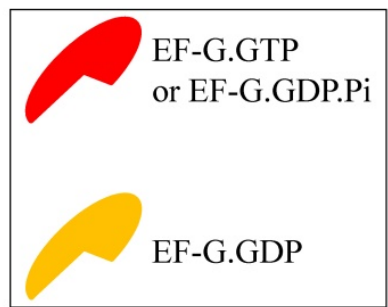

(d)

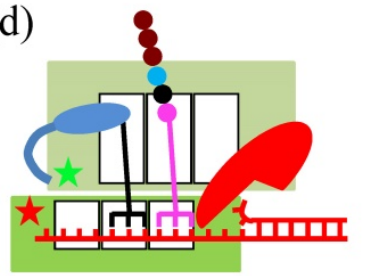

State $\mathbf{H} 2$ (c)
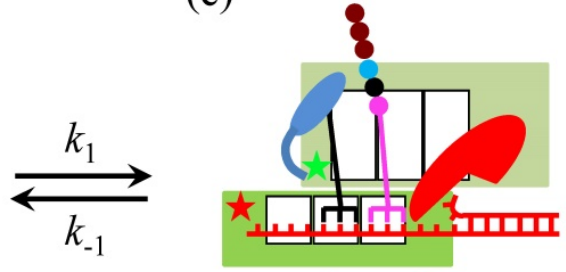

State HR $k_{2} \downarrow$ Ribosomal unlocking

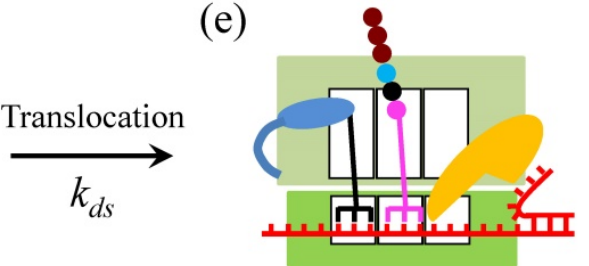

State POST2

Figure 4. Schematic representation of Model IV. From the posttranslocation state (State POST1), after rapid deacylated tRNA dissociation, the binding of the ternary complex in the A/T state, GTP hydrolysis, Pi and EF-Tu.GDP releases, the accommodation of the aminoacyl-tRNA into its fully bound A/A state and then the peptidyl transfer, the ribosomal complex transits to the classical non-rotated pretranslocation state. Then, EF-G.GTP of saturating concentration binds immediately, facilitating the classical non-rotated pretranslocation state transition to hybrid state (State H1). For convenience, the rate of transitions from State POST1 through State $\mathrm{HI}$ is denoted by $k_{0}$. Before the ribosomal unlocking, the transitions between rotated State HI and hyper-rotated State HR can occur (in State HR the Ll stalk also contacts the acceptor end of the deacylated tRNA). In State H1, the ribosomal unlocking occurs (State H2). The subsequent reverse intersubunit rotation causes the small ribosomal $30 \mathrm{~S}$ subunit translocating forward by unwinding three mRNA base pairs, with State $\mathrm{H} 2$ transiting to State POST2, from which the next elongation cycle continues. Schematic of the Cy3-labeled protein L9 and Cy5-labeled protein S6 in the smFRET experiments of Qin et al. [13] is shown.

\section{Model IV}

With smFRET by monitoring efficiency of FRET between Cy3-labeled protein L9 and Cy5-labeled protein S6 (see Fig. 4) combined with small angle X-ray scattering, Qin et al. [13] observed recently that in the absence of EF-G the presence of the downstream mRNA stem loop can drive the ribosomal subunits into a hyper-rotated state, with the L1 stalk being in an open conformation. Based on these smFRET data [13], a model (called Model IV) (Fig. 4) can be proposed, where it is argued that the ribosomal unlocking occurs inefficiently in the hyper-rotated state (State HR) and thus the ribosomal unlocking can only occur efficiently in the hybrid state. In addition, it is argued that in the hyper-rotated state the L1 stalk, albeit in the open conformation, still contacts the acceptor end of the deacylated tRNA, as proposed before [14]. In Model IV, the reduction of the translation rate arises from the entrance into State HR.

Besides being consistent with the smFRET data of Qin et al. [13], State HR in Model IV could also correspond to the non-canonical rotated state reported by Chen et al. [15]. However, because in State HR the L1 stalk still contacts the acceptor end of the deacylated tRNA, Model IV indicates that when translation through the mRNA secondary structure, at saturating EF-G.GTP the L1-tRNA FRET efficiency shows the high value $(\sim 0.8)$ only once before undergoing mRNA translocation, which is inconsistent with the smFRET data of Kim et al. [5]. Thus, Model IV is also not a reasonable one to describe the mechanism of ribosome translation through the mRNA secondary structure.

\section{Model V}

Another model (called Model V), which is similar to Model IV, can be proposed, as shown in Fig. 5. The only difference between Model V and Model IV is in the hyper-rotated state (State HR): although the L1 stalk is in the open conformation in State HR in both models, the L1 stalk in Model IV contacts whereas in Model V does not contact the acceptor end of the deacylated tRNA.

It is evident that Model $\mathrm{V}$ can give an explanation of the SmFRET data showing that when translation through the mRNA containing a downstream stem loop, even at saturating EF-G.GTP the L1-tRNA FRET efficiency showed multiple fluctuations between the low and high values. The period of high FRET efficiency at saturating EF-G 
corresponds to that after the EF-G-facilitated transition to State $\mathrm{H} 1$ but before the transition of State $\mathrm{H} 1$ to State HR, with the lifetime of $\tau_{\text {high }} \approx 2.1 \mathrm{~s}$ [5]; the period of low FRET efficiency corresponds to that after transition to State HR but before transition back to State H1, with the lifetime of $\tau_{\text {low }} \approx 0.55 \mathrm{~s}$ [5]; and the period of the last high FRET efficiency corresponds to that after the transition of State HR to State $\mathrm{H} 1$ to State $\mathrm{H} 2$ and before the transition of State $\mathrm{H} 2$ to State POST2, with the lifetime of $\tau_{\text {last }} \approx 6.4 \mathrm{~s}$ [5]. When translation through the single-stranded mRNA, no transition to State HR can occur, and thus the L1-tRNA FRET efficiency shows the high value only once before undergoing mRNA translocation. For this case, the period of the high or last FRET efficiency corresponds to that after the EF-G-facilitated transition to State $\mathrm{H} 1$ to State $\mathrm{H} 2$ but before the transition of State $\mathrm{H} 2$ to State POST, with the lifetime

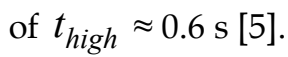

From Fig. 5, we can easily obtain the mean translation rate, $v$, having the form

$$
v^{-1}=\frac{1}{k_{0}}+\left(1+K_{1}\right) \frac{1}{k_{2}}+\frac{1}{k_{d s}}
$$

where $K_{1}=k_{1} / k_{-1}$. From the smFRET data of $\tau_{\text {high }} \approx 2.1 \mathrm{~s}$ and $\tau_{\text {low }} \approx 0.55 \mathrm{~s} \mathrm{[5],} \mathrm{we} \mathrm{have}$ $K_{1}=k_{1} / k_{-1}=\tau_{\text {low }} / \tau_{\text {high }} \approx 0.26$. Thus, from Eq. (1) we note that the entrance into State HR induced by the downstream duplex only has a slight effect on the reduction of the translation rate relative to that without the downstream duplex (with $K_{1} \approx 0$ ), especially under the conditions of $k_{0}<k_{2}$. Thus, it is expected that the observed reduction of the rate of translation through the mRNA duplex should arise mainly from the reduction of the rate constant of the mRNA translocation step. The above explanation of the smFRET data of $\tau_{\text {last }}$ being about 3-fold larger than $t_{\text {high }}$ [5] also implies that the reduction of the rate constant of the mRNA translocation step should have a large effect on the translation rate, as Model I showed. As studies showed [11], based on the consideration that the reduced translation rate arises mainly from the reduction of the rate constant of the mRNA translocation step, the theoretical data on dwell-time distribution of translation through the mRNA duplex versus the external force to unwind the duplex are deviated significantly away from the available smOT data [2]. Moreover, from the smFRET data showing that about $50 \%$ of the traces for the mRNA containing the stem loop were fluctuating at saturating concentration of EF-G.GTP [5], we have $k_{1} \approx$ $k_{2}$. The smFRET data of $\tau_{\text {high }} \approx 2.1 \mathrm{~s}$ gives $k_{1} \approx 0.48 \mathrm{~s}^{-1}$. Thus, the rate constant of ribosomal unlocking, $k_{2} \approx$ $0.48 \mathrm{~s}^{-1}$, which is also deviated significantly away from the available biochemical data of about $35 \mathrm{~s}^{-1}$ [16]. Consequently, Model V is also less likely.

\section{Model V}

(a)

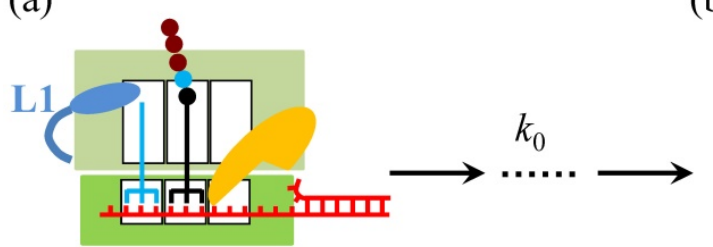

State POST1 (b)

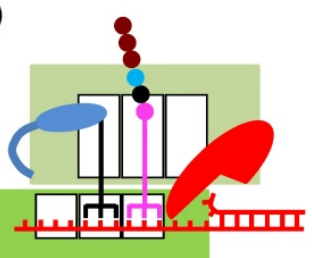

State H1

$\left.k_{2}\right\rfloor$ Ribosomal unlocking

(d)

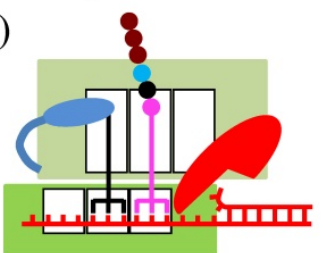

State $\mathrm{H} 2$ (c)
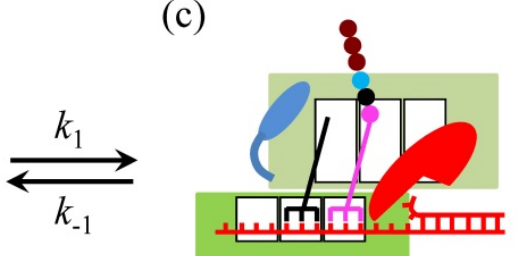

State HR

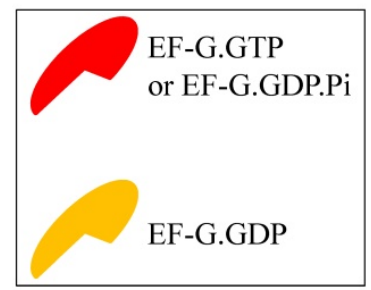

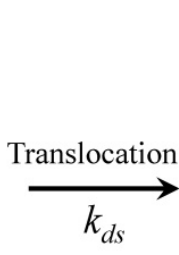

$k_{d s}$ (e)

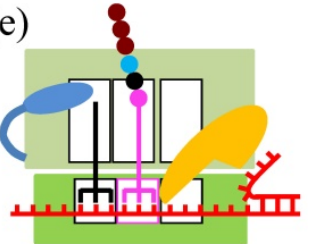

State POST2

Figure 5. Schematic representation of Model V. Model V is similar to Model IV, with the only difference between the two models is that in State HR the LI stalk in Model IV contacts whereas in Model $V$ does not contact the acceptor end of the deacylated tRNA. 


\section{Model VI}

Another model for ribosome translation through the mRNA duplex (called Model VI) is shown in Fig. 6 [7], which was proposed on the consideration that the $50 S \mathrm{E}$ and $\mathrm{P}$ sites have finite affinities to deacylated tRNA and peptidyl-tRNA, respectively $[17,18]$. When translation through the single-stranded mRNA, after the ribosomal unlocking occurs in the hybrid state, widening the mRNA channel in the 305 subunit [19-23], the rapid reverse ribosomal rotation causes two tRNAs coupled with mRNA to move from the $30 \mathrm{~S} P$ and A sites to $30 \mathrm{~S} \mathrm{E}$ and P sites, while the two tRNAs are kept fixed to the $50 \mathrm{~S} \mathrm{E}$ and $\mathrm{P}$ sites by their large affinity $\left(\Delta G_{E P}^{(50 S)}\right)$ for the two tRNAs (see, e.g., Ref. [24] for a more detailed pathway of ribosomal rotations causing ribosomal translocation). When translation through the mRNA duplex, after the ribosomal unlocking in the hybrid state the reverse ribosomal rotation would exert a force on the non-template strand of the closed duplex, because the diameter of the mRNA entry channel in the $30 \mathrm{~S}$ subunit is too small to accommodate the dimensions of the mRNA duplex [19-23,25]. The force drives unwinding of the mRNA duplex. The unwinding gives an energy barrier of $\Delta G_{b p}$ to impede the forward translocation of the $30 \mathrm{~S}$ subunit along the
mRNA, where $\Delta G_{b p}$ is the free-energy change of breaking three mRNA base pairs. Considering $\Delta G_{b p}$ comparable to $\Delta G_{E P}^{(50 S)}$, the reverse ribosomal rotation would cause either the movement of two tRNAs coupled with mRNA from the 30S P and A sites to $30 \mathrm{~S} E$ and $P$ sites while the two tRNAs are fixed to the 50S E and P sites (transition from State H2 to State POST2) or the movement of two tRNAs from the 50S E and P sites to 50S P and A sites while the two tRNAs coupled with mRNA are fixed to the $30 S \mathrm{P}$ and A sites (transition from State $\mathrm{H} 2$ to State F). As structural data showed [26], EF-G is kept in elongated form in State POST2 (the non-rotated posttranslocation state) and becomes compact in State F (a non-rotated pretranslocation state). The former transition corresponds to the effective translocation by unwinding three mRNA base pairs, while the latter transition corresponds to the futile translocation, with no mRNA base pair being unwound. After transition to the non-rotated conformation (both State POST2 and State F), the mRNA channel becomes tight again [27, 28]. From State F, after EF-G.GDP release, EF-G.GTP binding and forward intersubunit rotation, the next cycle of transition from State $\mathrm{H} 1$ to State $\mathrm{H} 2$ to State POST2 or transition from State H1 to State H2 to State F proceeds.

\section{Model VI}

(a)

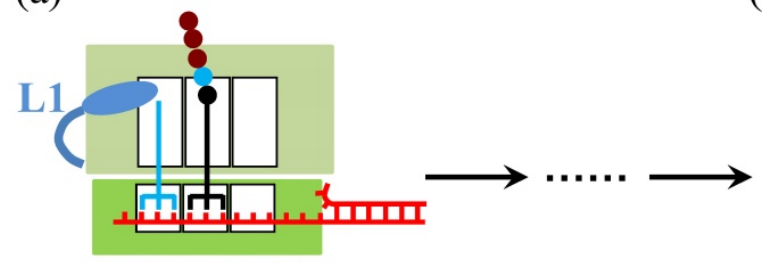

State POST1

(d)

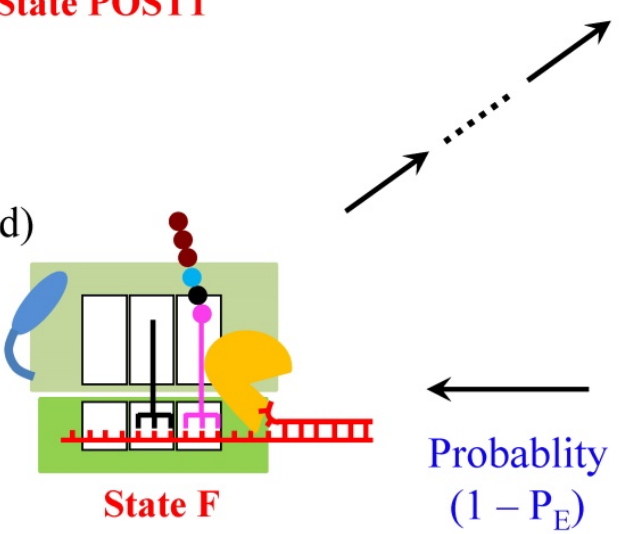

(b)

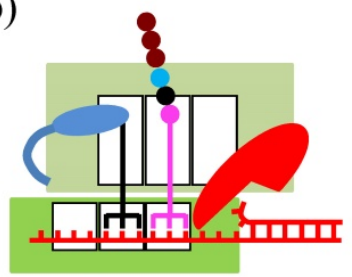

State H1 $\downarrow$ Ribosomal unlocking

(c)

State $\mathrm{H} 2$

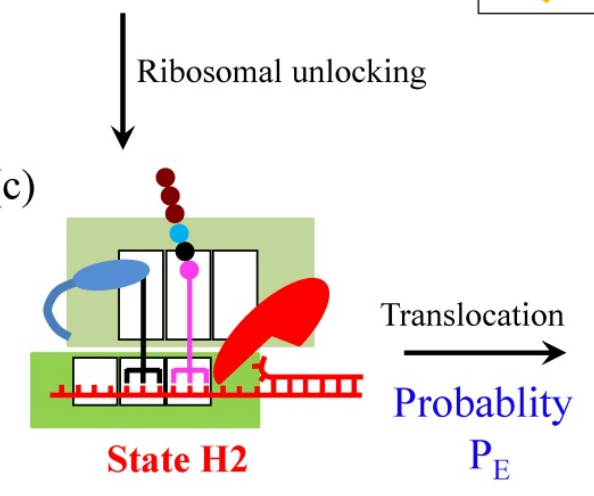

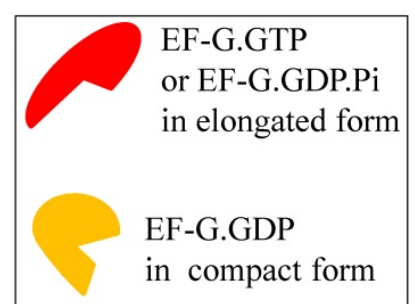

in compact form

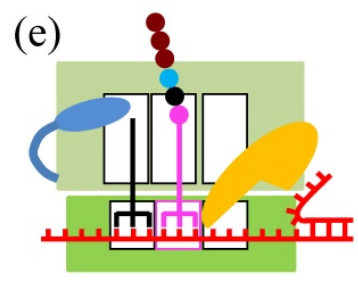

State POST2

Figure 6. Schematic representation of Model VI [7]. The transitions from State POST1 through State HI are the same as those in Fig. 4. In State HI, the ribosomal unlocking occurs (State H2). Then, the rapid reverse intersubunit rotation that is facilitated by the ribosomal unlocking causes either the effective translocation (from c to e) or the futile translocation (from c to d), becoming State POST2 and State F, respectively. The effective translocation induces unwinding of the mRNA duplex by the mechanical unwinding mechanism. The futile translocation disrupts the interactions of the $50 \mathrm{~S} E$ and $P$ sites with deacylated tRNA and the peptidyl-tRNA, respectively. From State F, after EF-G.GDP release, EF-G.GTP binding and forward intersubunit rotation, the next cycle of transition from (b) to (c) to (e) or transition from (b) to (c) to (d) proceeds. 
In Model VI, the ribosome uses only one active mechanism - the mechanical unwinding mechanism-for its helicase activity [7], consistent with the recent experimental evidence [12], and the reduction of the translation rate arises from the occurrence of the futile translocation rather than the reduction of the rate constant of the mRNA translocation step. The occurrence of the futile translocation is consistent with the proposal of futile elongation factor 2 (eEF2) cycling during ribosomal translocation in $80 \mathrm{~S}$ ribosome with the presence of mRNA secondary structures by Flanagan et al. [29]. With Model VI various available single-molecule experimental data on translation through the mRNA secondary structures can be explained quantitatively. For example, the smOT data of Qu et al. [3] showing a sigmoid dependence of the translation rate through the mRNA duplex on the unzipping force were reproduced well $[7,11]$, the theoretical data [11] on the distribution of dwell times under different unzipping force were in good agreement with the smOT data [2], the smFRET data by Chen et al. [4] on the effect of downstream mRNA secondary structures on tRNA translocation in the 50S subunit and deacylated tRNA dissociation from the E/E site were explained well [30], and moreover, the smFRET data by Kim et al. [5] on multiple fluctuations between the low and high values of the L1-tRNA FRET efficiency before undergoing mRNA translocation were quantitatively explained [10]. The model is also consistent with the
smFRET data showing that the downstream duplex has little effect on the time duration between the delivery of the ternary complex and the first transition to the high $(\sim 0.8)$ L1-tRNA FRET efficiency [5]. Thus, Model VI is a reasonable one to describe the mechanism of ribosome translation through the mRNA secondary structure.

\section{Model VII}

Since in the smOT experiments of Qu et al. [3] the ribosome translates patches of the same codons, another model (called Model VII) (Fig. 7), which is similar to Model VI, can also explain the smOT data [3]. In Model VII, after the ribosomal unlocking (State $\mathrm{H} 2$ ), the reverse ribosomal rotation would also cause either effective translocation (transition to State POST2) or futile translocation (transition to State F). However, the futile translocation in Model VII is realized by breaking the base-pairing interactions of the deacylated-tRNA and peptidyl-tRNA anticodons with the $30 \mathrm{~S} \mathrm{P}$ - and A-site codons, respectively, and then forming base-pairing interactions with the $30 \mathrm{~S} \mathrm{E}$ and P-site codons, respectively, while keeping the two tRNAs fixed to the 50S E and P sites. As in Model VI, the reduction of the translation rate in Model VII arises from the occurrence of the futile translocation rather than the reduction of the rate constant of the mRNA translocation step.

\section{Model VII}

(a)

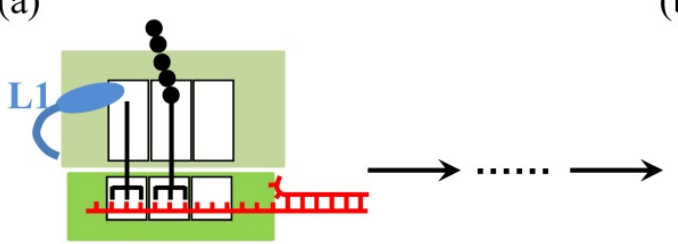

State POST1

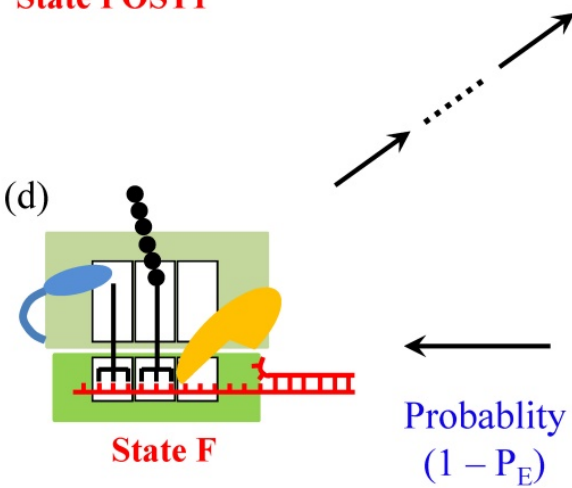

(b)

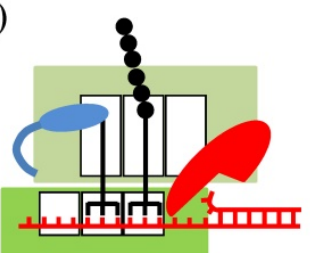

State H1 Ribosomal unlocking

(c)

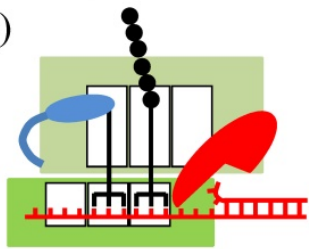

State $\mathbf{H} 2$

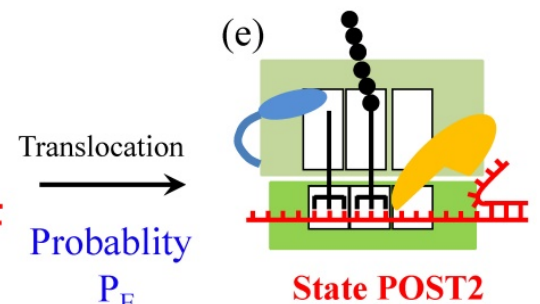

Figure 7. Schematic representation of Model VII. The model is only applicable to the case of translation of the same codons. The transitions from State POSTI through State $\mathrm{HI}$ are the same as those in Fig. 4 or Fig. 6. In State $\mathrm{H1}$, the ribosomal unlocking occurs (State $\mathrm{H} 2)$. Then, the rapid reverse intersubunit rotation that is facilitated by the ribosomal unlocking induces either the effective translocation (from c to e) or the futile translocation (from c to d), becoming State POST2 and State F, respectively. The effective translocation induces unwinding of the mRNA duplex by the mechanical unwinding mechanism. The futile translocation induces breaking the base-pairing interactions of deacylated-tRNA and peptidyl-tRNA anticodons with the 30S P- and A-site codons, respectively, and then forming base-pairing interactions with the 30S E and P-site codons, respectively. Note that State F is the same as State POST1. 


\section{Model VIII}

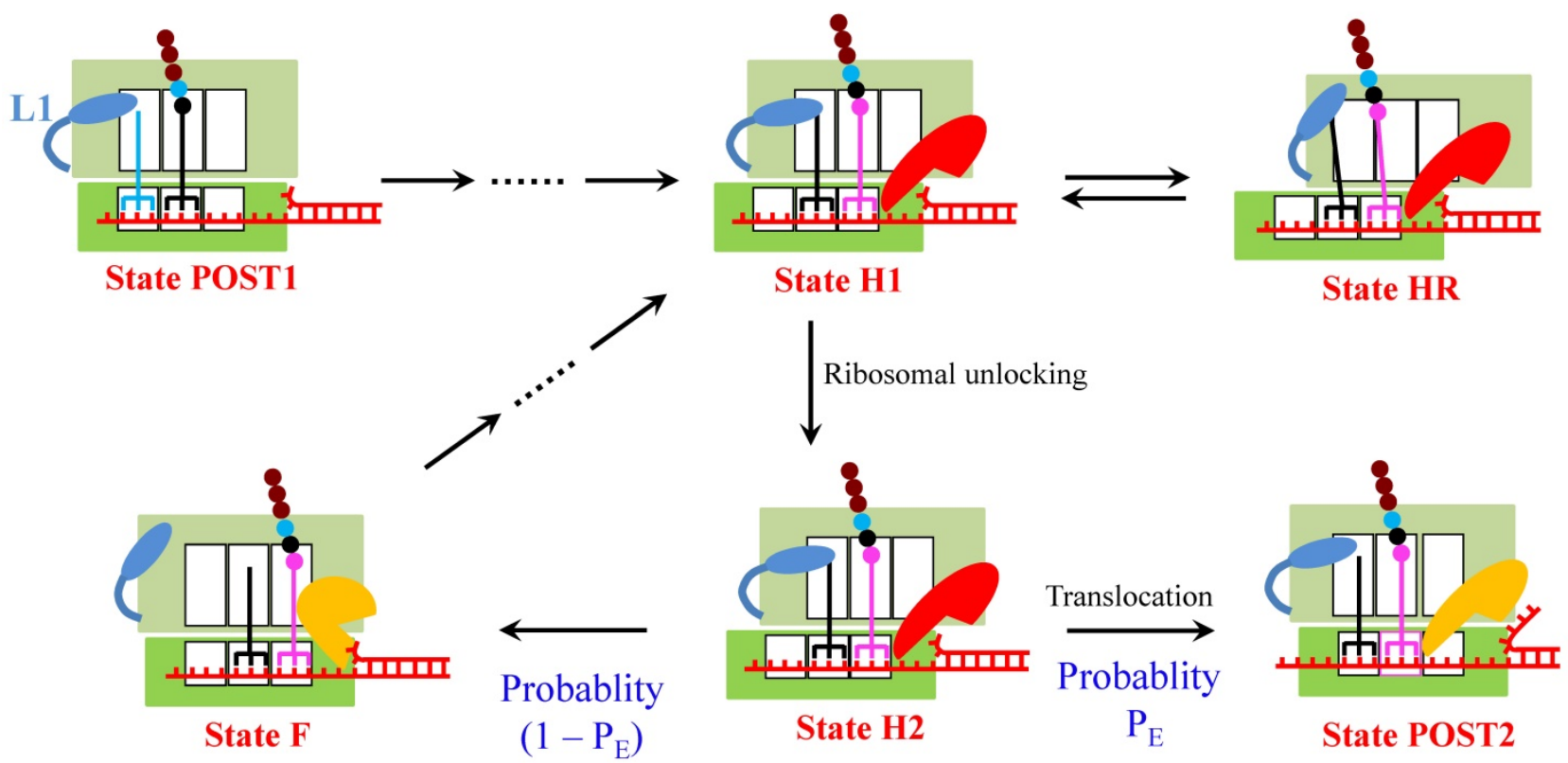

Figure 8. Schematic representation of Model VIII. The model is a combination of Model VI and Model IV.

It is important to note that Model VII is only applicable to the case when the three codons in the $30 \mathrm{~S}$ E, P and A sites are the same, but it not applicable to the case when the three codons are not the same. Moreover, even if the model could be applicable to the latter case, since both State F and State POST2 are the posttranslocation state the rate constant of deacylated tRNA dissociation from the E/E site of State F would be similar to that from the E/E site of State POST2. On the other hand, for both cases with and without the downstream secondary structure the mean times for transition from State POST1 to State POST2 are similar, since the transitions from State $\mathrm{H} 2$ to State POST2 for both cases are much faster than other transitions. Thus, the presence of the downstream secondary structure has nearly no effect on the rate of tRNA dissociation, which is inconsistent with the smFRET data of Chen et al. [4] showing that the presence of the downstream secondary structure decreases the rate of tRNA dissociation. Therefore, Model VII is not a reasonable one.

\section{Model VIII}

As discussed above, of Model I, Model II, Model III, Model IV, Model V, Model VI and Model VII, Model VI is the most reasonable one to describe the mechanism of ribosome translation through the mRNA secondary structure. Since under some conditions the presence of the downstream mRNA stem loop can drive the ribosomal subunits into the hyper-rotated state [13], a model (called Model VIII) can be proposed by combining Model VI and Model
IV, as shown in Fig. 8. As it is noted, in Model VIII the multiple fluctuations between the low and high values of the L1-tRNA FRET efficiency before undergoing mRNA translocation arise also from the occurrence of the futile translocation, as in Model VI. Apart from that the occurrence of the futile translocation makes the main contribution to the reduction of translation rate, the entrance into State HR also makes a minor contribution. Thus, Model VIII can still be consistent with various single molecule experimental data. Consequently, Model VIII is also applicable.

Similarly, by combining Model VI and Model V another model (called Model VIII') can be proposed. In Model VIII', the multiple fluctuations between the low and high values of the L1-tRNA FRET efficiency before undergoing mRNA translocation arise from both the occurrence of the futile translocation and the entrance into State HR. Apart from that the occurrence of the futile translocation makes the main contribution to the reduction of translation rate, the entrance into State HR also makes a minor contribution.

\section{Concluding remarks and perspectives}

Here, we review the three models (Model II, Model III and Model VI) for mRNA unwinding by the ribosome that have been proposed up to now and propose five new models (Model I, Model IV, Model V, Model VII and Model VIII/Model VIII'). We show that the theoretical results obtained based on Model I, Model II, Model III, Model IV, Model V and Model 
VII are not consistent with the available smFRET or smOT data. By contrast, it is shown that Model VI and Model VIII/Model VIII' can explain quantitatively diverse single-molecule experimental data. Thus, Model VI and Model VIII/Model VIII' are the most reasonable ones to describe the mRNA unwinding mechanism by the ribosome. In these three models, the ribosome uses the mechanical unwinding mechanism for its helicase activity. It is interesting to note that DNA helicases may share the similar mechanism of DNA unwinding [31, 32].

In order to further test Model VI and Model VIII/Model VIII', we suggest using other smFRET assays. (i) Instead of using Cy3-labeled L1 stalk and Cy5-labeled tRNA, as done by Kim et al. [5], one can use Cy3-labeled protein L9 and Cy5-labeled protein S6, as done by Cornish and his coworkers [13, 33], to visualize directly the multiple fluctuations between the classical non-rotated and rotated or hybrid states before undergoing translocation at saturating EF-G.GTP. With this kind of S6(Cy5)/L9(Cy3) labeled ribosomes, we can determine definitely whether the multiple fluctuations result from the transitions between the classical non-rotated and hybrid states, as Model VI and Model VIII (Figs. 6 and 8) show, or result from the transitions between the rotated/hybrid and hyper-rotated states, as Model V (Fig. 5) shows. (ii) Using Cy3-labeled ribosomal protein L11 and Cy5-labeled peptidyl-tRNA, as done in Chen et al. [4] and in Adio et al. [34], to visualize directly the multiple fluctuations between the hybrid state with the peptidyl-tRNA in the A/P site and the classical non-rotated pretranslocation state with the peptidyl-tRNA in the A/A site before undergoing translocation at saturating EF-G.GTP. (iii) Using Cy5-labeled L1 stalk and Cy3-labeled protein L33, as done by Cornish et al. [35], to visualize directly the multiple fluctuations of the L1 stalk between the open and closed conformations before undergoing translocation at saturating EF-G.GTP. (iv) Since in Model VI and Model VIII/Model VIII' multiple times of ribosomal unlocking occur before undergoing mRNA translocation and one ribosomal unlocking involves one round of the $30 \mathrm{~S}$ head rotations relative to the 30 S body [19-24, 36], one can label 305 head protein S13 with Cy 5 and large $50 \mathrm{~S}$ subunit protein L5 with Cy3, as done by Wasserman et al. [37], to visualize directly multiple rounds of the $30 \mathrm{~S}$ head rotations before undergoing mRNA translocation at saturating EF-G.GTP. (v) Furthermore, one can simultaneously label above components of the ribosomal complex as well as label the $3^{\prime}$ end of the mRNA with a fluorescence reporter, as usually done in the literature [38-43], and thus observe simultaneously the multiple intersubunit rotations, multiple 305 head rotations, multiple positional changes of the peptidyl-tRNA between A/P and A/A sites and multiple fluctuations of the L1 stalk between open and closed conformations before undergoing mRNA translocation at saturating EF-G.GTP.

\section{Acknowledgments}

This work was supported by the National Natural Science Foundation of China (Grant No. 11374352).

\section{Competing Interests}

The authors have declared that no competing interest exists.

\section{References}

[1] Takyar S, Hickerson RP, Noller HF. mRNA helicase activity of the ribosome. Cell. 2005; 120: 49-58.

[2] Wen J-D, Lancaster L, Hodges C, Zeri A-C, Yoshimura SH, Noller HF, Bustamante C, Tinoco Jr I. Following translation by single ribosomes one codon at a time. Nature. 2008; 452: 598-603.

[3] Qu X, Wen J-D, Lancaster L, Noller HF, Bustamante C, Tinoco Jr I. The ribosomeuses two activemechanisms to unwind messenger RNA during translation. Nature. 2011; 475: 118-121.

[4] Chen C, Zhang H, Broitman SL, Reiche M, Farrell I, Cooperman BS, Goldman YE. Dynamics of translation by single ribosomes through mRNA secondary structures. Nat Struct Mol Biol. 2013; 20: 582-588.

[5] Kim H-K, Liu F, Fei J, Bustamante C, Gonzalez Jr RL, Tinoco Jr I. A frameshifting stimulatory stem loop destabilizes the hybrid state and impedes ribosomal translocation. Proc Natl Acad Sci USA. 2014; 111: 5538-5543.

[6] Yao L, Li Y, Tsai T-W, Xu S, Wang Y. Noninvasive measurement of the mechanical force generated by motor protein EF-G during ribosome translocation. Angew Chem Int Ed. 2013; 52: 14041 -14044.

[7] Xie P. Model of ribosome translation and mRNA unwinding. Eur Biophys J. 2013; 42: 347-354.

[8] Shakiba B, Dayeri M, Mohammad-Rafiee F. Modeling of ribosome dynamics on a ds-mRNA under an external load. J Chem Phys. 2016; 145: 025101.

[9] Uemura S, Aitken CE, Korlach J, Flusberg BA, Turner SW, Puglisi JD. Real-time tRNA transit on single translating ribosomes at codon resolution. Nature. 2010; 464: 1012-1017.

[10] Xie P. Origin of multiple intersubunit rotations before EF-G-catalyzed ribosomal translocation through the mRNA with a downstream secondary structure. BMC Biophys. 2014; 7: 12

[11] Xie P. Dwell-time distribution, long pausing and arrest of single-ribosome translation through the mRNA duplex. Int J Mol Sci. 2015; 16: 23723-23744.

[12] Zhong Z, Yang L, Zhang H, Shi J, Vandana JJ, Lam DTUH, Olsthoorn RCL, Lu L, Chen G. Mechanical unfolding kinetics of the SRV-1 gag-pro mRNA pseudoknot: possible implications for -1 ribosomal frameshifting stimulation. Sci Rep. 2016; 6: 39549.

[13] Qin P, Yu D, Zuo X, Cornish PV. Structured mRNA induces the ribosome into a hyper-rotated state. EMBO Rep. 2014; 15: 185-190.

[14] Xie P. Origins of biphasic kinetics of ribosomal translocation. Unpublished.

[15] Chen J, Petrov A, Johansson M, Tsai A, O'Leary SE, Puglisi JD. Dynamic pathways of -1 translational frameshifting. Nature. 2014; 512: 328-332.

[16] Savelsbergh A, Katunin VI, Mohr D, Peske F, Rodnina MV, Wintermeyer W. An elongation factor G-induced ribosome rearrangement precedes tRNA-mRNA translocation. Mol Cell. 2003; 11: 1517-1523.

[17] Lill R, Robertson JM, Wintermeyer W. Binding of the 30-terminus of tRNA to $23 \mathrm{~S}$ rRNA in the ribosomal exit site actively promotes translocation. EMBO J. 1989; 8: 3933-3938.

[18] Feinberg JS, Joseph S. Identification of molecular interactions between P-site tRNA and the ribosome essential for translocation. Proc Natl Acad Sci USA. 2001; 98: 11120-11125.

[19] Ratje AH, Loerke J, Mikolajka A, et al. Head swivel on the ribosome facilitates translocation by means of intra-subunit tRNA hybrid sites. Nature. 2010; 468: 713-716.

[20] Ramrath DJ, Lancaster L, Sprink T, Mielke T, Loerke J, Noller HF, Spahn CMT. Visualization of two transfer RNAs trapped in transit during elongation factor G-mediated translocation. Proc Natl Acad Sci USA. 2013; 110: 20964-20969.

[21] Zhou J, Lancaster L, Donohue JP, Noller HF. Crystal structures of EF-G-ribosome complexes trapped in intermediate states of translocation. Science. 2013; 340: 1236086.

[22] Zhou J, Lancaster L, Donohue JP, Noller HF. How the ribosome hands the A-site tRNA to the P site during EF-G-catalyzed translocation. Science. 2014; 345: 1188-1191. 
[23] Nguyen K, Whitford PC. Steric interactions lead to collective tilting motion in the ribosome during mRNA-tRNA translocation. Nature Commun. 2016; 7: 10586.

[24] Xie P. On the pathway of ribosomal translocation. Int J Biol Macromol. 2016; 92: 401-415

[25] Yusupova GZ, Yusupov MM, Cate JH, Noller HF. The path of messenger RNA through the ribosome. Cell. 2001; 106: 233-241.

[26] Lin J, Gagnon MG, Bulkley D, Steitz TA. Conformational changes of elongation factor $\mathrm{G}$ on the ribosome during tRNA translocation. Cell. 2015; 160: 219-227.

[27] Xie P. Model of ribosomal translocation coupled with intra- and inter-subunit rotations. Biochem Biophys Rep. 2015; 2: 87-93.

[28] Frank J, Agrawal RK. A ratchet-like inter-subunit reorganization of the ribosome during translocation. Nature. 2000; 406: 318-322.

[29] Flanagan IV JF, Namy O, Brierley I, Gilbert RJC. Direct observation of distinct A/P hybrid-state tRNAs in translocating ribosomes. Structure. 2010; 18: 257-264.

[30] Xie P. Dynamics of tRNA translocation, mRNA translocation and tRNA dissociation during ribosome translation through mRNA secondary structures. Eur Biophys J. 2014; 43: 229-240.

[31] Xie P. A unified model of nucleic acid unwinding by the ribosome and the hexameric and monomeric DNA helicases. J Theor Biol. 2015; 380: 359-366.

[32] Xie P. Dynamics of monomeric and hexameric helicases. Biophys Chem. 2016; 211: 49-58.

[33] Cornish PV, Ermolenko DN, Noller HF, Ha T. Spontaneous intersubunit rotation in single ribosomes. Mol. Cell. 2008; 30: 578-588.

[34] Adio S, Senyushkina T, Peske F, Fischer N, Wintermeyer W, Rodnina MV. Fluctuations between multiple EF-G-induced chimeric tRNA states during translocation on the ribosome. Nature Commun. 2015; 6: 7442.

[35] Cornish PV, Ermolenko DN, Staple DW, Hoang L, Hickerson RP, Noller HF, Ha T. Following movement of the L1 stalk between three functional states in single ribosomes. Proc Natl Acad Sci USA. 2009; 106: 2571 - 2576.

[36] Xie P. Dynamic relationships between ribosomal conformational and RNA positional changes during ribosomal translocation. Heliyon. 2016; 2: e00214.

[37] Wasserman MR, Alejo JL, Altman RB, Blanchard SC. Multiperspective SMFRET reveals rate-determining late intermediates of ribosomal translocation. Nat Struct Mol Biol. 2016; 23: 333-341.

[38] Walker SE, Shoji S, Pan D, Cooperman BS, Fredrick K. Role of hybrid tRNA-binding states in ribosomal translocation. Proc Natl Acad Sci USA. 2008; 105: 9192-9197.

[39] Peske F, Savelsbergh A, Katunin VI, Rodnina MV, Wintermeyer W. Conformational changes of the small ribosomal subunit during elongation factor G-dependent tRNA-mRNA translocation. J Mol Biol. 2004; 343: 1183-1194.

[40] Shi X, Chiu K, Ghosh S, Joseph S. Bases in 16S rRNA important for subunit association, tRNA binding, and translocation. Biochemistry. 2009; 48: 6772-6782.

[41] Liu Q. Fredrick K. Contribution of intersubunit bridges to the energy barrier of ribosomal translocation. Nucleic Acids Res. 2013; 41: 565-574.

[42] Ermolenko DN, Noller HF. mRNA translocation occurs during the second step of ribosomal intersubunit rotation. Nat Struct Mol Biol. 2011; 18: 457-463.

[43] Guo Z, Noller HF. Rotation of the head of the 30S ribosomal subunit during mRNA translocation. Proc Natl Acad Sci USA. 2012; 109: 20391-20394. 